\title{
To Build an Optimization Model of Multi-Factor Decision and a Study of Its Application
}

\author{
Xiao Xiaonan \\ Xiamen University Tan Kah Kee College \\ Zhangzhou Fujian China \\ xiaoxn@xujc.com
}

\begin{abstract}
By using the fuzzy transformation and fuzzy integral, this article digs into how to conduct best fuzzy multidimensional synthetic decision theoretically and practically, and also finds out the mathematical model of the best fuzzy multidimensional synthetic decision so as to raise the standard of synthetic decision and enlarge the sphere of its applications in natural science and social science. In this article, the author gives the examples of scientific management of the education quality, reasonable assignment of graduate students and medical diagnosis. These modern examples of the best decision indicate that the best fuzzy multidimensional synthetic decision widely used in practice as a good tool must lead to satisfactory results.
\end{abstract}

Keywords-optimal multidimensional synthetic decision; majorized model; fuzzy transformation; reliability analysis

\section{INTRODUCTION}

With the development of modern science, its emphasis now shifts more and more rapidly form the research on the definite object by the method of analysis to the research on the indefinite object by the method of synthesis. After every concrete science has the typical phenomenon in its own sphere fully studied, it is now engaged in enlarging scope sphere and is ready to make the research on the untypical phenomenon of the both. The trend of the penetrating between the different natural science, between the different social science, and between the social science and natural science appears apparently with the time going by. The former branch-bound line is broken and the frontier science springs up. The fuzzy mathematics appearing in 1960's is a great breakthrough in the prolongation of mathematics research.

We can use fuzzy mathematics to study the fuzzy phenomenon in the objective world, so the limitation of the tradition mathematics has been smoothed away and many problems which can't be solved by tradition mathematics have been solved. And fuzzy mathematics applies a good situation to the mathematization of natural science, social science, system science, idea science and body science. In recent years, many achievements concerning the fuzzy mathematics have appeared, but fuzzy decision theory is now in its childhood. So this article tries to conduct the research on the best fuzzy multidimensional synthetic decision and enlarge the sphere in which the best fuzzy multidimensional synthetic decision can be applied[1].

\section{RESEARCH ON BFMSD}

Many system in the world are influenced by many factors as well as have many aims, this is so-called "Multidimensional Synthetic Decision". Generally a decision problem is always coupled with fuzziness, randomness experience characteristics.

So it is common to adopt the fuzzy synthetic decision. The following would lay the Emphasis on this problem.

In order to evaluate something synthetically, we must pay attention to the following three factors :

Collection of elements $U=\left\{u_{1}, u_{2}, \cdots, u_{n}\right\}$

$u_{i}$ shows the factor that has to be considered by something;

Decision collection $V=\left\{v_{1}, v_{2}, \cdots, v_{m}\right\}$

$v_{i}$ shows the stage of decision;

Single elements decision, it is a fuzzy mapping from $U \rightarrow V$. According to the

Fuzzy mapping law, a fuzzy mapping ${ }_{\sim}^{f}$ can decide a

fuzzy relation $\underset{\sim}{R} \underset{\sim}{f}$, it can be expressed by a fuzzy matrix $\underset{\sim}{R \in M_{n \times m}}$. So $\underset{\sim}{R}$ can be considered as a fuzzy transformation from $U \rightarrow V$.

Thus an evaluation space (U, V, ${ }_{\sim}^{R}$ )may make up a model of synthetic evaluation.

Suppose a fuzzy subset in $\mathrm{U}$

$\stackrel{A}{\sim}\left(a_{1}, a_{2}, \cdots, a_{n}\right)$, in which $a_{i}$ represents the weighted number and it satisfies

$$
\sum_{i=1}^{n} a_{i}=1 .
$$

In the given fuzzy transformation $\underset{\sim}{R}$ and factor weight $\underset{\sim}{A}$, we can get a fuzzy subset from the fuzzy relations composite operation, that is : 
$\underset{\sim}{A} R_{\sim}{ }_{\sim}^{B} \in M_{l \times m}$

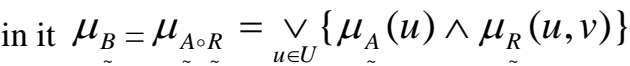

The aforementioned is a mathematical model of fuzzy synthetic evaluation. In fact, the fuzzy synthetic evaluation is using the known invert image (weight matrix) and mapping (one-factor evaluation matrix) to get the result of synthetic evaluations.

In additional, we can use fuzzy integrate to form a sort of the model of the synthetic evaluation.

Suppose $U=\left\{u_{1}, u_{2}, \cdots, u_{n}\right\}$ is a collection consisting of $n$ factors. $P(U)$ is

U' s preparing field. We have the given fuzzy vector on $\mathrm{U}$

$M=\left(m_{1}, m_{2}, \cdots, m_{n}\right)$

Expresses the "chief factor". It is actually the leaders' evaluations on the

importance of $n$ factors.

Every state on $U$, there's $H \in F(U)$

$H=\left(h_{1}, h_{2}, \cdots, h_{n}\right)$

$\mathrm{H}$ about "chief factor"-M's synthetical evaluation is :

$$
\int_{u} H(u) \circ \prod(\bullet)=H \circ M=\underset{k=1}{\vee}\left(h_{k} \wedge m_{k}\right)
$$

The aforementioned possibility measure and fuzzy integral theory solve the rationality of the evaluation model[2].

In order to avoid the above model's defects caused by omitting the sub-important factor, we can pretreat the evaluation. For example, we can give a bottom line to every factor's satisfactory degree, when one object's satisfactory degree is below the standard, we can drive the object off the evaluation. Today, Fuzzy $\mathrm{N}$-integrate enlarges operator sphere of fuzzy integrate, and on the base of $(\vee, \wedge)$ some new operators appear.

We can use every sort of synthetic evaluation models resulting from different fuzzy integrate to deal with some different practical questions.

Suppose the fuzzy Vector $M$ which stands for the possibility measures is fixed. There are $\mathrm{m}$ evaluators to evaluate the given object a individually, then

$$
H_{j}=\left(h_{j 1}, h_{j 2}, \cdots, h_{j n}\right) \in F(U)
$$

stands for the satisfactory evaluation on $\alpha$ of Evaluator Number $\mathrm{j}, j=1,2, \cdots, m$.

And to the fixed factor $u_{i} \in U$

$$
H_{1}\left(u_{i}\right)=h_{1 i}, H_{2}\left(u_{i}\right)=h_{2 i}, \cdots, H_{m}\left(u_{i}\right)=h_{m i}
$$

Can be considered as sample value whose volume is $\mathrm{m}$ resulting from motherbody $U_{i} . U_{i}$ stands for the random variable of the objective evaluation of $\alpha$ on factor $u_{i}$. If you consider the social evaluation on $u_{i}$ true, then the aforementioned abstraction is reasonable. From the strong law of large number in probability theory

$$
p\left\{\lim _{m \rightarrow \infty} \frac{1}{m} \sum_{j=1}^{m} H_{j}\left(u_{i}\right)=h_{i}\right\}=1
$$

$h_{i}$ is the mathematical expectation of the random variable $U_{i 1}$ which stands for the social evaluation on the base of social outlook on value. If we let $\mathrm{i}$ run from 1 to $\mathrm{n}$, then we get $H \in F(U)$,

$$
H=\left(h_{1}, h_{2}, \cdots, h_{n}\right)
$$

Stands the social evaluation of the satisfaction on $\alpha$. We can't get $\mathrm{H}$ directly, but we can get the sample value $H_{1}, H_{2}, \cdots, H_{m}$ mentioned above and then we have

$$
p\left\{\lim _{m \rightarrow \infty} \frac{1}{m} \sum_{j=1}^{m} H_{j}=H\right\}=1
$$

According to the convergence theorem of fuzzy integrate series to the fuzzy integrate function $\mathrm{H}, H_{n}, n=1,2, \cdots$, on the finite field $U$, If

$$
\lim _{m \rightarrow \infty} H_{n}=H
$$

Then we can get

$$
\lim _{m \rightarrow \infty} \int_{U} H_{n}(u) \circ \prod(\bullet)=\int_{U} H(u) \circ \prod(\bullet)
$$

That's also $E_{0}=\int_{U} H(u) \circ \prod(\bullet)=H_{0} M, E_{0}$ is the social synthetic evaluation to $\alpha$.For the same reason, $E_{j}=\int_{U} H_{j}(u) \circ \prod(\bullet)=H_{j} \circ M \quad$ is the synthetical evaluation of the evaluator number i on $\alpha, j=1,2, \cdots, m$.

Let's combine (2) with (3) , then we can get

$P\left\{\lim _{m \rightarrow \infty} \int_{U} \frac{1}{m} \sum_{j=1}^{m} H_{j}(u) \circ \prod(\bullet)=\int_{U} H(u) \circ \prod(\bullet)\right\}=1$

In other words, when $\mathrm{m}$ is very big, according to probability 1

$\int_{U} \frac{1}{m} \sum_{j=1}^{m} H_{j}(u) \circ \prod(\bullet)=E_{0}$

Attention, the fuzzy integral doesn't fit the common addition of function to the distributive(aw) thus, in general, we say

$$
\frac{1}{m} \sum_{j=1}^{m} \int_{U} H_{j}(u) \circ \prod(\bullet) \neq \int_{U} \frac{1}{m} \sum_{j=1}^{m} H_{j}(u) \circ \prod(\bullet)
$$


That is to say, we cannot use $\frac{1}{m} \sum_{j=1}^{m} E_{j}$ to evaluate $E_{0}$.

We call $E_{j}$ the individual evaluation, $j=1,2, \cdots, m$, and call $E_{0}$ the group evaluation true value, while $\int_{U} \frac{1}{m} \sum_{j=1}^{m} H_{j}(u) \circ \prod(\bullet)$ is called a group evaluation whose volume is $m$, we write it down as $\hat{E}(m) \cdot \hat{E}(m)$ as an approximation of $E_{0}$, is fairer than any other $E_{i}[3 \sim 5]$.

\section{APPLICATION EXAMPLES}

The job assignment of the college is rather complicated, which touches upon a lot of objects and factors. How to use the talented persons rationally is the crux in the job assignment. So the author has applied the fuzzy multidimensional synthetic decision method to change the qualitative analysis of the job assignment of the graduates into quantitative analysis, which shows a best mathematical model of the job assignment and offers a reliable scientific basis.

The fuzzy multidimensional synthetic decision can be widely used in every field in natural science and social science[7]. For example, the consultative system of the medical experts and the computer interrogation, which marks the modernization in medical diagnosis, is an excellent achievement in scientific research that combines the fuzzy multidimensional synthetic decision with practice. The fundamental procedure in diagnosis is:

$$
\begin{aligned}
& \text { (symptom) (diagnosis) (diagnosis) }
\end{aligned}
$$

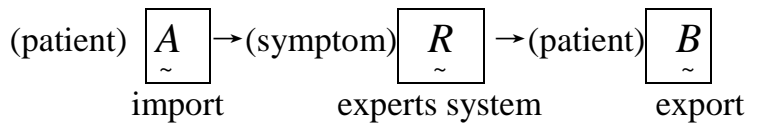

The main model:

The exerts' experience in treating or curing the patients $\rightarrow$ mathematicism $\rightarrow$ computer study $\rightarrow$ feedback revise $\rightarrow$ consultative system of experts $\rightarrow$ computer interrogation.
It is not difficult to realize "the sensor interrogating" with the help of the fuzzy transformation and computers. In fact, in our country, the fuzzy mathematical model that Doctor Guang Youbo uses to cure liver diseases has been made into the software of the expert system, and the computer interrogation has been realized successfully.

The best fuzzy multidimensional synthetic decision is an optimization. This method is often used in solving the problem with multiple targets and factors, which is difficult to evaluate, but it can be settled by the fuzzy transformation[8]. If we can program the computer and realize the automation of the evaluation, the effect will be still better. In addition, if we can choose the proper factors and show the weighted numbers of every factor, and if those who take part in evaluation possess representative and practical experience, there is very important significance in improving the effect of the fuzzy multidimensional synthetic decision.

\section{REFERENCES}

[1] Qiu J; Zhang L.F-interfere law genercetion and its feature recognition[J]. Jourmal of Systems Engineering and Electronics, 2009, 20(4):777-783.

[2] Wang Q H.Statistical estimation in partial linear models with covariate data missing at random[J].Annals of the Institute of Statistical Mathematics,2009,61:47-84

[3] Saaty T. Exploring the interface between Hierachies Multiple Objectives and Fuzzy Sets[J]. Fuzzy and Systems, 1978,(1): 57 68

[4] Xiao Xiaonan.Best efficiency unbiased estimation of several kinds of essential random truncated distribution functions parameter in reliable analys is of existence and life[J]. Joural of Mthematical Study, 2010, 43(4): 342-351.

[5] Yu L, Chen G D, Chu J. Optimal guaranteed cost control of linear uncertain systems: LMI approach[J]. IFAC, 1999, G-2e-21-1: 541 546.

[6] Gong Guanglu. Introduction of stochastic differential equations[M]. Beijin: Peking University Press,1995:396-432

[7] Anderson T W. An Introduction to Multivariate Statistical Aualysis[M]. New York: John Wiley \& Sons, 1990.232 296.

[8] Chen F.D, Li Z,Huang Y.J.. Note on the permanence of a competitive system with infinite delay and feedback controls[J].Nonlinear Anal. Real World Appl., 2007,8(2):680-687. 